\title{
A Review of Efficiency Improvement Techniques in Modern Communication Systems
}

\begin{abstract}
Peddi Saurabh
Department of Electronics and

Communication Engineering

RV College Of Engineering

Bangalore

Email: peddisaurabh.ec17@rvce.edu.in

Abstract-This paper discusses Doherty Power Amplifier(DPA) and its evolution over the years. The basic operational principle of the Doherty amplifier and its defective behavior that has been originated by transistor characteristics will be presented.The different research trends, all aimed to improve the advantages of the Doherty scheme and to solve its inherent drawbacks, are discussed.
\end{abstract}

\author{
Poornima Asuti \\ Department of Electronics and \\ Communication Engineering \\ RV College Of Engineering \\ Bangalore
}

\author{
Prof. Deepika P \\ Department of Electronics and \\ Communication Engineering \\ RV College Of Engineering \\ Bangalore
}

Index Terms-doherty power amplifiers, load pull, matching networks

\section{INTRODUCTION}

To meet ever increasing demands for higher data rates and larger capacity, new modulation schemes have been developed and wider frequency bands, e.g., at mmwave, have been designated to 5G. Massive multi-input multi-output (MIMO), that uses a large number of antennas at the transmitter and receiver, has been considered as one of the key enabling technologies in $5 \mathrm{G}$ to improve data throughput and spectrum efficiency [3]. These new application scenarios pose stringent requirements on the wireless transceiver frontends and call for special considerations at both circuit and system design levels. In the transmitter, power amplifiers (PAs) should accommodate complex modulated signals, featuring high peak-to-average power ratio (PAPR) and wide modulation bandwidth. [1].

The DPA basically consists of two amplifiers having their output power combined through a load modulation network. It can maintain high efficiency over a large power range and it features a simple circuit implementation compared to the other architectures. [2]. A Matching network should be designed to satisfy all the requirements.

Unfortunately, the classical DPA suffers intrinsic bandwidth limitations, mainly due to narrow-band quarter-wavelength transmissions lines used for impedance transformation. Bandwidth extension is thus an important consideration in modern DPA designs and it has received increasing attention in recent research, especially for wideband 5G applications [3]. The letter is organised as follows. The importance and evolution of DPA is discussed in Section II. In Section III theory and design methodology of the DPA is explained. Section IV summarises the review.

\section{IMPORTANCE AND EVOLUTION OF DPA}

The back-off efficiency enhancement issue in modern communication systems can be approached with alternative strategies, like outphasing, bias modulation and envelope tracking. The outphasing PA adopts two saturated, high efficiency amplifiers, fed with constant envelope signals, conveniently obtained from the input signal. Adopting the Chireix power combiner, signal amplification can be performed with high total efficiency, but it degrades the PA linearity. Recently, the outphasing amplifier has been progressed significantly.

The bias modulation technique, or the Kahn transmitter in its analog form, also adopts a saturated high efficiency PA, usually a switching current amplifier, driven by a constant envelope signal carrying the phase information of the input signal. The amplitude information is recovered by modulating the drain voltage of the PA with a linear envelope amplifier. A more viable solution is the envelope tracking where a linear PA, driven with the amplitude modulated input signal, is adopted and the drain bias voltage tracks the envelope of the signal to minimize the power dissipation. Also in this case, when a large modulation bandwidth is required, the envelope amplifier becomes critical, and solutions need to be found to trade-off bandwidth and efficiency. Although the envelope tracking has become very popular for handset PAs, its employment as a class $\mathrm{AB}$ replacement for base stations is not straightforward due to the high power involved.

Considering this aspect, DPAs are often preferred because they can be effectively considered as class AB PA replacements. In fact, the other described techniques should refer to the whole transmitters rather than to the PA. In this context, they require a deep revision of the complete system architecture, with a strong impact on back compatibility and cost. Shows that one of the major concerns about DPA is its limited RF bandwidth. However, as discussed later in this paper, different solutions have been proposed to solve this issue. Also the other techniques have been revised to overcome their intrinsic limitations: for example, outphasing PAs can now achieve good average efficiency. Other issues remain open, such as modulation bandwidth in high power outphasing and envelope 
tracking, and DPAs are still a preferred choice.

The importance of DPA for the microwave research community can be highlighted by the fact that, browsing the IEEE electronic library in the considered time span, among 1100 results on "microwave power amplifiers" and "RF power amplifiers", more than 100 relate to DPAs. What it is interesting to notice is that, along the years, the number of published papers on PAs and, in particular, DPAs, has constantly grown, following the spreading of high data rate mobile communications, . Furthermore, the impact ratio between papers on DPA and total papers on PAs is constantly increasing.

\section{THEORY AND DESIGN METHODOLOGY OF THE DPA}

The schematic of DPA is shown in Fig.1 The RF circuit

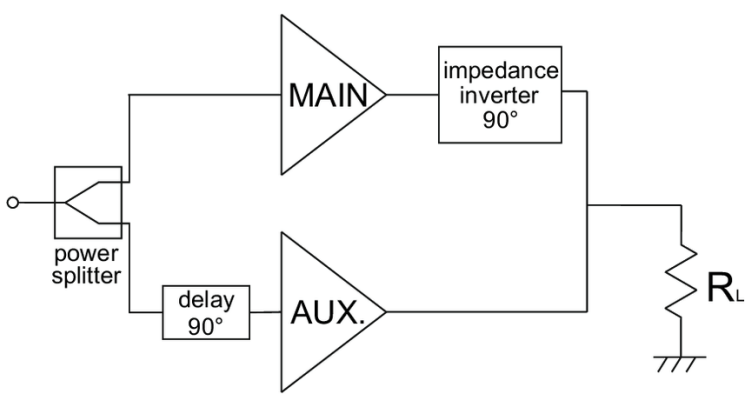

Fig. 1: Schematic of Doherty Power Amplifier

design of a Doherty amplifier uses a main or carrier amplifier that is typically biased for Class $\mathrm{AB}$ operation. A second active device, typically named the auxiliary or peaking amplifier that is normally biased for Class $\mathrm{C}$ operation.

The signal enters the overall Doherty power amplifier and is presented to a splitter. This creates two signals that are phase shifted by $90^{\circ}$ with respect to each other. The reason for this is that inductive splitters are used to reduce the power loss and these create a $90^{\circ}$ phase shift between the two signals. One output is presented to the carrier amplifier. This is designed to accommodate the lower power levels encountered around the average power level. This is designed to provide optimum efficiency for these power levels.

The signal is also presented to the peaking amplifier. This is biased so that it only started operating when large peaks are present that the carrier amplifier would not be able to accommodate on its own. Being a higher power amplifier, this would not provide high levels of efficiency for the lower power levels and therefore it only operates when higher power levels are present. In this way optimum efficiency is obtained over the power range.

One the signal has passed through the RF amplifier circuits themselves, the outputs are combined using a reverse of the splitter circuit. As this also has a $90^{\circ}$ phase shift, this is used to counteract the phase shift at the input. As a result, signals from the two amplifier sections remain in phase. The efficiency of Doherty over class B in compared in Fig.2

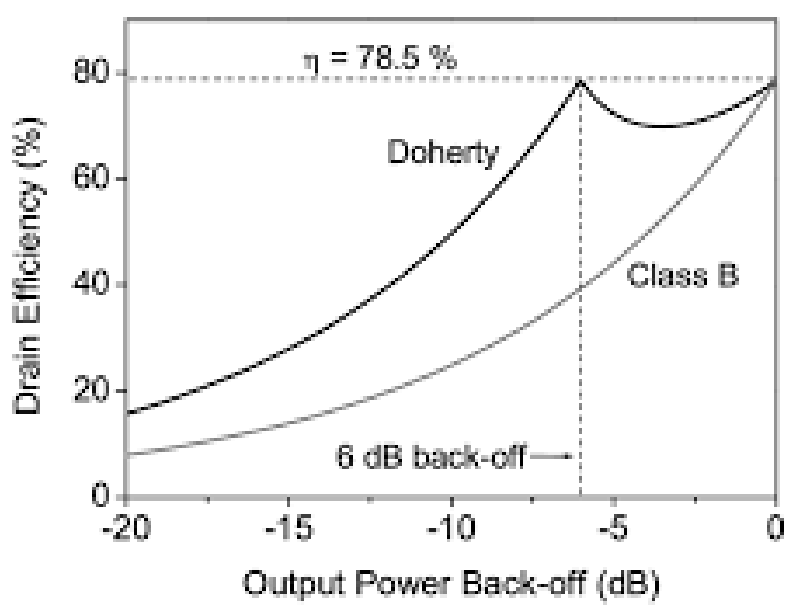

Fig. 2: Efficiency of Doherty over Class B amplifier as a function of output power

The impedance inverter at the output of the main stage is necessary to guarantee the correct load modulation, and the phase difference that it introduces is recovered at the DPA input inserting a delay line. Main and auxiliary output matching networks present Ropt to the main and auxiliary devices at saturation, as in standard PAs. The so-called offset lines can be inserted in the output section of the DPA. Their characteristic impedance is chosen equal to the load seen by the stage at saturation, in order not to affect the saturated DPA behaviour, but they are useful to optimize performance at lower power. In fact, for the main stage, the offset line length is tuned to provide a purely real 2Ropt load at the device drain, a condition not generally satisfied with a simple matching network. The auxiliary stage offset line ensures instead that the turned-off auxiliary does not influence the load seen by the main stage. As a result, offset lines improve DPA performance, e.g., the gain flatness vs. output power, since they allow to optimize the load modulation. The DPA ideal efficiency vs. is represented in Fig. Fig. 3 The ideal efficiencies of a 3-stage DPA, a 4-way DPA, and an ideal tuned load class B are also reported. The multi-way DPAs can be considered as DPAs where the auxiliary is a combined PA: on monolithic solutions, larger periphery is usually adopted for the auxiliary stage (asymmetrical devices). In Fig. 3, the power distributions (approximated with Rayleigh distributions) of widely employed modulated signals for modern wireless communications are also represented. It can be clearly seen that the 2-way DPA advantages in terms of efficiency are maximized at $6 \mathrm{~dB} \mathrm{OBO}$, and are still significant for larger PAPR signals. Moreover, efficiency can be further increased introducing more sophisticated DPA solutions. 


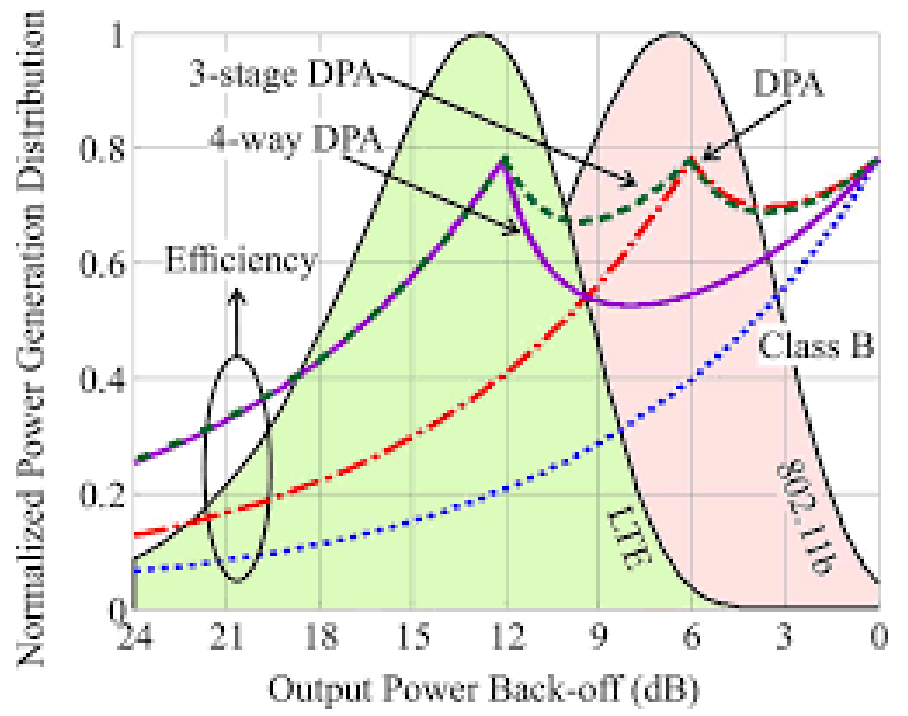

Fig. 3: Normalized efficiency of class B and Doherty PAs (standard, 4-way and 3-stage) vs. normalized output power, over-imposed to examples ofnormalized power distributions of modulated signals for wireless applications.

\section{CONCLUSION}

Different amplifier typologies and techniques that improve efficiency at higher backoff of the modern communication systems have been discussed. Doherty Power Amplifier topology is compared with others and the theory and design of the same has been explained. Design of output match and offset lines and their relevance in improving DPA bandwith has been discussed. Further enhancements, such as 3-way and other nway DPAs and their significance in improving efficiency has been presented.

\section{REFERENCES}

[1] R. Q. S. J. Y. C. V. Camarchia, M. Pirola and B. Kim, "The doherty power amplifier: Review of recent solutions and trends," IEEE Transactions on Microwave Theory and Techniques, vol. 63, no. 2, pp. 559-571, Feb 2015.

[2] J. C. D. P. Nguyen and A. Pham, "A doherty amplifier with modified load modulation scheme based on load-pull data," IEEE Transactions on Microwave Theory and Techniques, vol. 66, no. 1, pp. 227-236, Jan 2018.

[3] Z. D. C. H. J. P. J. Pang, S. He and F. You, "Design of a post-matching asymmetric doherty power amplifier for broadband applications," IEEE Microwave and Wireless Components Letters, vol. 26, no. 1, pp. 52-54, Jan 2016. 